\title{
A study on the Electrolytes Concentration and Lipid Profile in Cardiac and Cardiovascular defective patients
}

\author{
Malathi $\mathbf{R}^{1^{*}}$ \\ ${ }^{1 *}$ Corresponding author, Department of Life Sciences, Kristu Jayanti College - Autonomous.
}

\begin{abstract}
Abstrac
Cardiovascular diseases are common all over the world, and atherosclerosis of coronary arteries is considered to be the leading cause of premature death among men. Serum electrolyte concentrations after myocardial revascularization varied within normal ranges. Magnesium sulphate infusion did not decrease the rate of postoperative atrial fibrillation during the early postoperative period in normomagnesemic patients. The main risk factors for Cardiovascular diseases are age, male sex, hypercholesterolemia [especially in case of cholesterol with low density lipoproteins (LDL)], smoking, systemic hypertension, and diabetes mellitus. The aim of the study was to compare the serum electrolytes $\left[\mathrm{Na}^{+}, \mathrm{K}^{+}\right.$, $\mathrm{Cl}^{-}$and $\mathrm{Ca}^{2+}$ concentration and lipid profile[cholesterol, triglycerides, HDL, LDL, VLDL,LDL/HDL ratio and cholesterol/HDL ratio] of normal persons with cardiac and cardiovascular defective patients. Sodium concentration of cardiac and cardiovascular defective patients varied within normal ranges. Potassium concentration of cardiac and cardiovascular defective patients was very low, when compared with the normal persons. Chloride concentration of Cardiac and cardiovascular defective patients varied within the normal ranges. Calcium concentration of cardiac and cardiovascular defective patients was very high, when compared with the normal persons. Cholesterol, HDL, VLDL, LDL, LDL/HDL ratio, cholesterol/HDL ratio varied within the normal ranges. Triglycerides concentration gets fluctuated and got increased in few cardiac and cardiovascular defective patients, when compared with normal persons.
\end{abstract}

Keywords Electrolytes, Cardiac and cardiovascular disease, HDL, VLDL, LDL, HDL

\section{Introduction}

Heart failure is a key cause of cardiovascular mortality and morbidity, resulting in more than one million hospitalizations per year in the USA, and it is the most common hospital discharge diagnosis among subjects older than 65 years (Caterina Urso et al., 2015). Several mechanisms interact to produce electrolyte and lipid profile changes. The reduction in cardiac output leads directly to a reduction in renal blood flow, with impairment of renal excretion of water and electrolytes, and it causes the activation of several neuro-hormonal responses which affect both cardiovascular homeostasis and electrolyte balance (Dargie H J, 1990).

This study was designed to assess the changes of serum electrolytes $\left[\mathrm{Na}^{+}, \mathrm{K}^{+}, \mathrm{Cl}^{-}\right.$and $\left.\mathrm{Ca}^{2+}\right]$ concentration and lipid [cholesterol, triglycerides, HDL,LDL,VLDL,LDL/ HDL ratio and cholesterol/HDL ratio] profile in different cardiac and cardiovascular problems such as RHD,CHD,CAD,MR,TR,MS etc., using normal persons as control.

\section{Materials and method}

\section{Chemicals:}

All the chemicals used for these experimental protocols were obtained from Lab-Care Diagnostics (India) PVT.LTD. Mumbai.

\section{Blood Sample:}

Cardiac and cardiovascular defective patients' blood samples were collected from Sailabs \# 5 to 9,Dharapuram road, Old Govt. Hospital Compound (East), Tirupur.

All the estimations were performed by the standard protocol. Estimation of calcium in serum by Colorimetric end point test method [Arsenazo III method], Estimation of chloride in serum by Colorimetric end point test method [mercurous (II) thiocyanate method], Estimation of potassium and sodium was done by colorimetric method, Estimation of Cholesterol by CHOD/PAP method[cholesterol oxidase peroxidase method], Estimation of Triglycerides by Colorimetric enzymatic test using glycerol-3-phosphate-oxidase[GPO], Estimation of High density lipoprotein-Cholesterol [HDL-C] by Clearance method, Determination of Very low density lipoprotein [VLDL], Low density lipoprotein [LDL], LDL/HDL ratio and cholesterol/HDL ratio by standard formulae.

Very low density lipoprotein $[\mathrm{VLDL}]=$ Triglycerides $\div 5$

Low density lipoprotein [LDL] = Cholesterol-HDL-VLDL

Cholesterol $/ \mathrm{HDL}$ ratio $=$ Cholesterol $\div \mathrm{HDL}$

$\mathrm{LDL} / \mathrm{HDL}$ ratio $=\mathrm{LDL} \div \mathrm{HDL}$

Statistical Analysis: The results were expressed as mean \pm SEM and comparisons between the 2 sets of data were made by using students $t$ - test and their significance were determined. 


\section{Result}

Table-1 represents, the electrolytes such as sodium $\left[\mathrm{Na}^{+}\right]$, potassium $\left[\mathrm{K}^{+}\right]$, chloride $[\mathrm{Cl}]$ and calcium $\left[\mathrm{Ca}^{2+}\right]$ concentration of the cardiac and cardiovascular defective patients.

Table-2 represents, the lipid profile [cholesterol, triglycerides, HDL, VLDL, LDL, LDL/HDL ratio and cholesterol/HDL ratio] of the cardiac and cardiovascular defective patients.Table-3 represents, the electrolytes such as sodium $\left[\mathrm{Na}^{+}\right]$, potassium $\left[\mathrm{K}^{+}\right]$, chloride $\left[\mathrm{Cl}^{-}\right]$and calcium $\left[\mathrm{Ca}^{2+}\right]$ concentration and lipid profile [cholesterol, triglycerides, HDL, VLDL, LDL, LDL/HDL ratio and cholesterol/HDL ratio] of both the normal persons and diseased subjects.

The cholesterol, HDL, VLDL, LDL, LDL/HDL ratio, cholesterol/HDL ratio varied within the normal ranges. Triglycerides concentration gets fluctuated and got increased in few cardiac and cardiovascular defective patients, when compared with normal persons. (Fig 2)

\section{Discussion}

A large body of research indicates that plasma cholesterol levels are important risk factors for coronary artery disease $[C A D]$.Lifestyle modification programs are known to improve cardiovascular risk profiles through significant reductions in LDL and total cholesterol. The lifestyle intervention also was effective in improving lipoprotein profiles that contribute to coronary artery disease risk (David Decewicz et al., 2009).

High plasma cholesterol is known to alter the cholesterol: phospholipids ratio in the plasma membrane, which leads to increased membrane viscosity. These changes facilitate monocyte adhesion and chemotaxis that precedes the development of fatty streaks (Bhagwat et al., 2009).

The close temporal association between the totally reversible conduction defect and hyponatremia strongly suggests that hyponatremia played a role in the pathogenesis of the conduction defect (Pawan Suri et al., 2009). Most of the studies, designed to evaluate changes in electrolyte levels after CPB, find enhanced electrolyte depletion and electrolyte balance disorders (Milda Svagzdiene et al., 2009). Congestive heart failure subjects progress multiple acid base and electrolyte defects due to several pathophysiological mechanisms. Their incidence is often correlated with the severity of cardiac dysfunction; additionally, these imbalances are associated with a poor prognosis (Caterina Urso et al., 2015).

\section{Conclusion}

Comparative study of cardiac and cardiovascular defective patients with normal individuals suggests that, sodium and chloride concentration of cardiac and cardiovascular defective patients varied within the
Table 1: Electrolytes concentration in Cardiac and Cardiovascular defective-patients.

\begin{tabular}{|c|c|c|c|c|c|c|}
\hline Subjects & Sex & Age(years) & $\mathrm{Na}[\mathrm{mEq} / \mathrm{l}]$ & $\begin{array}{c}\mathrm{K} \\
{[\mathrm{mmol} / \mathrm{l}]}\end{array}$ & $\mathrm{Cl}[\mathrm{mEq} / \mathrm{l}]$ & $\mathrm{Ca}[\mathrm{mg} / \mathrm{dl}]$ \\
\hline 1 & Female & 49 & 123 & 2.70 & 109.80 & 20.32 \\
\hline 2 & Female & 55 & 152 & 2.60 & 111.35 & 21.85 \\
\hline 3 & Female & 07 & 153 & 0.40 & 102.46 & 22.13 \\
\hline 4 & Male & 25 & 152 & 1.00 & 107.99 & 22.05 \\
\hline 5 & Female & 35 & 153 & 2.50 & 111.12 & 22.12 \\
\hline 6 & Male & 49 & 154 & 1.70 & 99.82 & 20.62 \\
\hline 7 & Female & 44 & 154 & 2.00 & 103.28 & 21.82 \\
\hline 8 & Female & 70 & 151 & 2.20 & 104.51 & 22.12 \\
\hline 9 & Female & 12 & 130 & 2.60 & 108.32 & 22.06 \\
\hline 10 & Male & 44 & 145 & 2.70 & 107.51 & 22.12 \\
\hline 11 & Female & 25 & 153 & 1.50 & 102.29 & 22.93 \\
\hline 12 & Female & 35 & 152 & 2.50 & 106.19 & 22.56 \\
\hline 13 & Male & 15 & 155 & 1.80 & 109.79 & 21.67 \\
\hline 14 & Female & 50 & 154 & 1.50 & 110.48 & 21.79 \\
\hline 15 & Male & 50 & 155 & 2.40 & 112.38 & 22.11 \\
\hline 16 & Male & 55 & 154 & 1.70 & 110.31 & 22.10 \\
\hline 17 & Male & 33 & 154 & 1.10 & 101.03 & 22.60 \\
\hline 18 & Female & 17 & 153 & 2.70 & 111.40 & 21.87 \\
\hline 19 & Female & 32 & 153 & 1.50 & 111.02 & 21.01 \\
\hline 20 & Male & 40 & 153 & 2.02 & 100.30 & 22.30 \\
\hline 21 & Female & 54 & 150 & 1.90 & 108.50 & 22.00 \\
\hline 22 & Female & 51 & 151 & 1.40 & 105.90 & 22.01 \\
\hline 23 & Male & 50 & 126 & 2.50 & 108.50 & 21.03 \\
\hline 24 & Male & 54 & 152 & 1.40 & 111.35 & 21.85 \\
\hline 25 & Female & 35 & 154 & 0.06 & 101.50 & 23.10 \\
\hline 26 & Male & 27 & 153 & 1.50 & 106.55 & 23.00 \\
\hline 27 & Male & 37 & 154 & 2.40 & 112.00 & 23.00 \\
\hline 28 & Female & 49 & 155.20 & 1.80 & 98.50 & 21.50 \\
\hline 29 & Female & 51 & 155 & 2.00 & 104.21 & 22.12 \\
\hline 30 & Female & 44 & 152 & 1.20 & 105.20 & 23.00 \\
\hline 31 & Female & 38 & 152 & 1.90 & 105.20 & 22.50 \\
\hline
\end{tabular}

All values represent Mean \pm SEM and its significance of all the parameters in diseased condition. 
Table 2 : Lipid profile in Cardiac and Cardiovascular defective patients

\begin{tabular}{|c|c|c|c|c|c|c|c|}
\hline Subjects & $\begin{array}{l}\text { Cholesterol } \\
{[\mathrm{mg} / \mathrm{dl}]}\end{array}$ & $\begin{array}{c}\text { Triglycerides } \\
{[\mathrm{mg} / \mathrm{dl}]}\end{array}$ & $\begin{array}{c}\text { HDL } \\
{[\mathrm{mg} / \mathrm{dl}]}\end{array}$ & $\begin{array}{l}\text { VLDL } \\
{[\mathrm{mg} / \mathrm{dl}]}\end{array}$ & $\begin{array}{c}\text { LDL } \\
{[\mathrm{mg} / \mathrm{dl}]}\end{array}$ & $\begin{array}{l}\text { LDL/HDL } \\
\text { ratio }\end{array}$ & $\begin{array}{l}\text { Cholesterol/ } \\
\text { HDL ratio }\end{array}$ \\
\hline 1 & 151.07 & 87.15 & 42.10 & 17.43 & 91.54 & 2.2 & 3.6 \\
\hline 2 & 150.91 & 108.43 & 43.16 & 21.69 & 86.06 & 1.9 & 3.5 \\
\hline 3 & 226.03 & 91.15 & 42.00 & 18.23 & 165.80 & 3.9 & 5.4 \\
\hline 4 & 149.83 & 152.21 & 39.16 & 30.44 & 80.23 & 2.0 & 3.8 \\
\hline 5 & 158.08 & 109.34 & 44.18 & 21.80 & 92.10 & 2.1 & 3.6 \\
\hline 6 & 169.28 & 113.91 & 44.10 & 22.80 & 102.38 & 2.3 & 3.8 \\
\hline 7 & 182.78 & 182.17 & 48.17 & 36.43 & 98.18 & 2.0 & 3.8 \\
\hline 8 & 191.15 & 103.98 & 40.16 & 20.79 & 130.20 & 3.2 & 4.75 \\
\hline 9 & 161.49 & 151.97 & 39.71 & 30.39 & 91.39 & 2.3 & 4.06 \\
\hline 10 & 101.23 & 123.00 & 39.18 & 24.60 & 37.45 & 0.96 & 2.60 \\
\hline 11 & 163.23 & 162.80 & 42.01 & 32.56 & 88.66 & 2.1 & 3.88 \\
\hline 12 & 145.42 & 193.75 & 49.12 & 38.75 & 57.55 & 1.2 & 2.96 \\
\hline 13 & 252.56 & 460.09 & 68.00 & 92.02 & 92.54 & 1.4 & 3.71 \\
\hline 14 & 113.44 & 96.23 & 41.81 & 19.25 & 52.38 & 1.3 & 2.71 \\
\hline 15 & 128.35 & 188.74 & 38.16 & 37.68 & 52.51 & 1.4 & 3.36 \\
\hline 16 & 112.23 & 104.15 & 36.12 & 20.83 & 55.28 & 1.5 & 3.11 \\
\hline 17 & 139.00 & 157.21 & 41.06 & 31.44 & 66.50 & 1.6 & 3.38 \\
\hline 18 & 161.15 & 94.13 & 40.18 & 18.83 & 102.14 & 2.5 & 4.01 \\
\hline 19 & 111.22 & 103.14 & 40.18 & 20.62 & 50.42 & 1.3 & 2.76 \\
\hline 20 & 138.00 & 156.30 & 40.05 & 31.26 & 66.69 & 1.7 & 3.45 \\
\hline 21 & 250.50 & 458.50 & 67.00 & 91.70 & 91.80 & 1.4 & 3.73 \\
\hline 22 & 145.00 & 193.50 & 48.12 & 38.70 & 58.18 & 1.2 & 3.01 \\
\hline 23 & 152.00 & 86.54 & 43.11 & 17.31 & 91.58 & 2.1 & 3.50 \\
\hline 24 & 149.50 & 109.00 & 44.17 & 21.80 & 83.53 & 1.9 & 3.38 \\
\hline 25 & 225.50 & 92.03 & 50.05 & 18.41 & 157.04 & 3.1 & 4.50 \\
\hline 26 & 150.50 & 153.03 & 40.01 & 30.61 & 79.88 & 1.9 & 3.76 \\
\hline 27 & 159.00 & 108.50 & 45.12 & 21.70 & 92.18 & 2.0 & 3.52 \\
\hline 28 & 170.12 & 114.50 & 45.20 & 22.90 & 102.02 & 2.3 & 3.76 \\
\hline 29 & 183.12 & 182.50 & 49.12 & 36.50 & 97.50 & 1.9 & 3.72 \\
\hline 30 & 192.20 & 104.10 & 40.50 & 20.82 & 130.88 & 3.2 & 4.75 \\
\hline 31 & 195.34 & 104.12 & 42.16 & 20.82 & 132.36 & 3.1 & 4.63 \\
\hline
\end{tabular}

All values represent Mean \pm SEM and its significance of all the parameters in diseased condition.

HDL=High Density Lipoprotein; VLDL=Very Low Density Lipoprotein; LDL=Low Density Lipoprotein
Table 3: Different Parameters In Both Diseased And Normal Samples

\begin{tabular}{|c|c|c|c|c|}
\hline S.No: & Parameters & $\begin{array}{c}\text { Diseased samples } \\
\text { Mean } \pm \text { SEM }\end{array}$ & $\begin{array}{c}\text { Normal samples } \\
\text { mean } \pm \text { SEM }\end{array}$ & t-Test \\
\hline 1 & Sodium $\left[\mathrm{Na}^{+}\right]$ & $150.23 \pm 1.45$ & $141.50 \pm 0.8791$ & $P>0.05$ \\
\hline 2 & Potassium $\left[\mathrm{K}^{+}\right]$ & $1.84 \pm 0.1177$ & $4.36 \pm 0.1125$ & $P>0.05$ \\
\hline 3 & Chloride $\left[\mathrm{Cl}^{-}\right]$ & $106.73 \pm 0.7227$ & $106.05 \pm 1.04$ & $P<0.05$ \\
\hline 4 & Calcium $\left[\mathrm{Ca}^{2+}\right]$ & $22.04 \pm 0.1176$ & $9.47 \pm 0.2691$ & $\mathrm{P}>0.05$ \\
\hline 5 & Cholesterol & $163.85 \pm 6.66$ & $164.60 \pm 3.06$ & $\mathrm{P}<0.05$ \\
\hline 6 & Triglycerides & $149.88 \pm 15.82$ & $71.60 \pm 4.45$ & $\mathrm{P}>0.05$ \\
\hline 7 & HDL & $44.30 \pm 1.25$ & $43.62 \pm 1.03$ & $\mathrm{P}<0.05$ \\
\hline 8 & VLDL & $29.97 \pm 3.16$ & $14.32 \pm 0.8886$ & $P>0.05$ \\
\hline 9 & LDL & $89.58 \pm 5.37$ & $106.18 \pm 3.48$ & $P>0.05$ \\
\hline 10 & LDL/HDL Ratio & $3.69 \pm 0.1111$ & $3.77 \pm 0.0445$ & $P<0.05$ \\
\hline 11 & $\begin{array}{l}\text { Cholesterol/HDL } \\
\text { Ratio }\end{array}$ & $2.03 \pm 0.1225$ & $2.44 \pm 0.0789$ & $P<0.05$ \\
\hline
\end{tabular}

HDL=High Density Lipoprotein; VLDL=Very Low Density Lipoprotein; LDL=Low Density

Lipoprotein. Each value represents the mean \pm SEM; $n=31$; Compared to control ' $P$ ' value and its significance were represented.

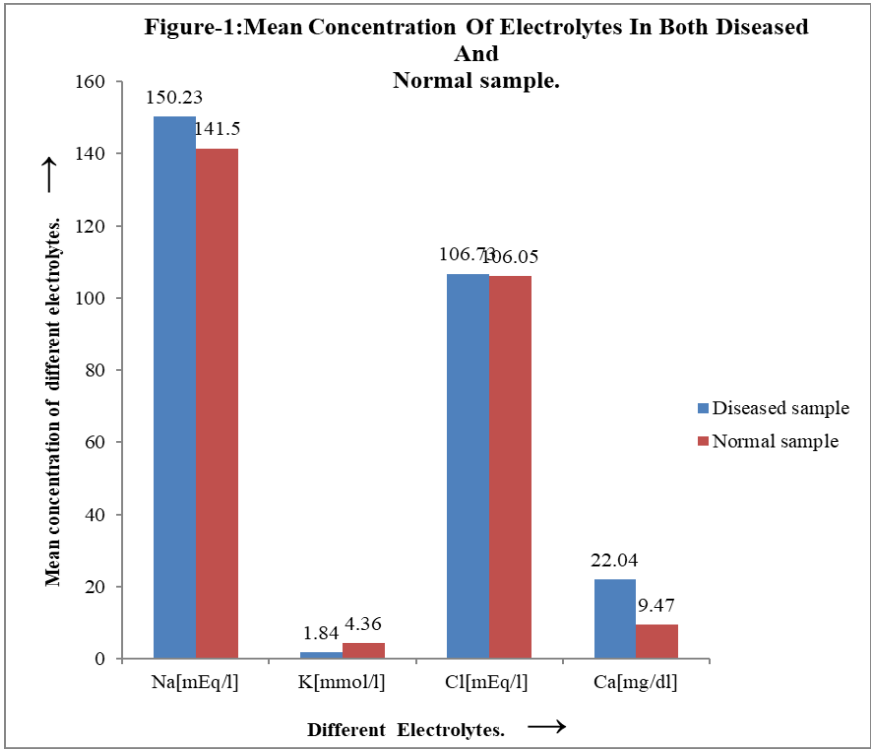

normal ranges. Potassium concentration of cardiac and cardiovascular defective patients was very low and calcium concentration of cardiac and cardiovascular defective patients was very high. Cholesterol, HDL, VLDL, LDL, LDL/HDL ratio and cholesterol/HDL ratio of cardiac and cardiovascular defective patients varied within the normal ranges. Triglycerides concentrations of the cardiac and cardiovascular defective patients get fluctuated and increased slightly. Thus, this study concluded that the electrolytes concentration of 


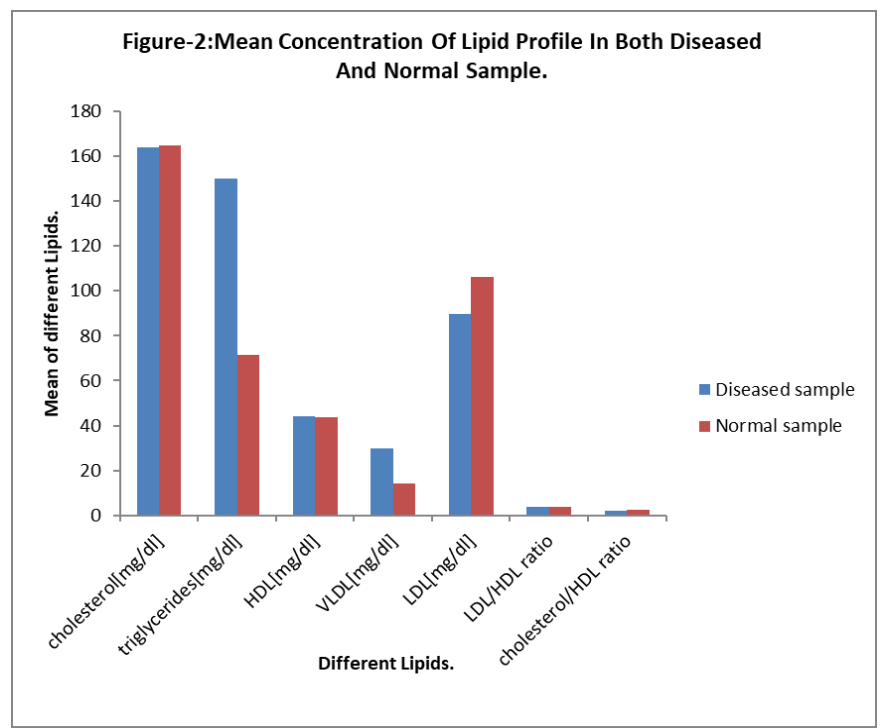

cardiac and cardiovascular defective patients varies when compared to the normal individuals. Cholesterol, HDL, VLDL, LDL, LDL/HDL ratio and cholesterol/HDL ratio do not have any change whereas triglyceride concentration of cardiac and cardiovascular defective patients increases slightly. Hence, there is a significant change in electrolytes concentration and considerable change in lipid profile of the cardiac and cardiovascular defective patients. This study can be further extended by analyzing a large number of samples with specific cardiac problems of interest.

\section{References}

1. David J. Decewicz, David M. Neatrour, Amy Burke, Marry Jane Haberkorn, Heather L. Patney, Marina N. Vernalis and Darrell L. Ellsworth: Effects of Cardiovascular lifestyle change on lipoprotein subclass profiles defined by nuclear magnetic resonance spectroscopy: Lipids in Health and disease 2009,8:26, doi:10.1186/1476-511X-8-26

2. Bhagwat V.R., Yadav A.S, Rathod I.M. Homocysteine, lipid indices and antioxidants in patients with ischaemic heart disease from Maharashtra, India. Singapore Med J 2009; 50(4): 418-424.

3. Pawan Suri .H.K., Alai M.S., Rather A.H. and Jalal S. Hyponatremia presenting as cardiac conduction defect. JK Sci, 2009, 11(2), 85-86.

4. Milda Svagzdiene, Edmundas Sirvinskas, Rimantas Benetis, Laima Raliene, and Violeta Simatoniene. "Atrial fibrillation and changes in serum and urinary electrolyte levels after coronary artery bypass grafting surgery." Medicina 45, 12 (2009): 960.

5. Caterina Urso, Brucculeri, S., \& Caimi, G. Acidbase and electrolyte abnormalities in heart failure: pathophysiology and implications. Heart failure reviews, 20(4),2015, 493-503. https://doi. org/10.1007/s10741-015-9482-y

6. Dargie H.J. Interrelation of electrolytes and reninangiotensin system in congestive heart failure. Am J Cardiol. 1990;65:28E-32E. rameters in diseased condition. 